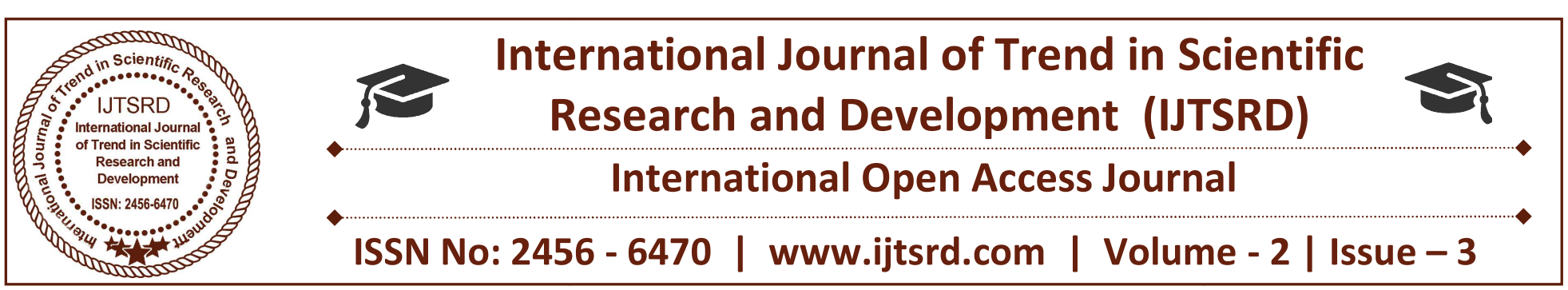

\title{
Arduino Controlled Robotic Arm
}

\author{
${ }^{1}$ K Aishwarya, ${ }^{2} \mathrm{~J}$ Priyadharshini, ${ }^{3} \mathrm{G}$ Rajeswari \\ ${ }^{1}$ Students, ${ }^{2}$ Assistant Professor, ${ }^{3}$ Head of the Department \\ ${ }^{1,2,3}$ Department of CSE, Sri Muthukumaran Institute of Technology, Chennai, Tamil Nadu, India
}

\section{ABSTRACT}

The evolution of Internet of Things has been tremendous in today's generation. A Promising and challenging mission is the use of autonomous robot systems to automate tasks in the field of maintenance. Major concern of evolution in robotics involves reducing human burden. This project work addresses the critical environment where Human presence is mandatory due to its nature of demanding accuracy like bomb diffusion, Chemical and radiation containment etc. A promising and challenging mission is the use of autonomous robot system to automate task in the field of maintenance. Each device is uniquely identifiable by the controlling software which is the core concept of Internet of Things Due to the remote operation procedure of a ROBOT .It lags in human precision and adaptability. This project proposes a novel idea of imitating the human hand movement with a virtually controlled robotic arm, which can be utilized in human endurance situations. A video and audio interface plug-in will be developed to recognize audio commands and also video gestures. Every method is experimentally validated and discussed.

Keywords: Internet of Things, Arduino, Relay, Sensors

\section{INTRODUCTION}

We know that in day to day life the work of humans has been tremendous. Most of the people are working on but at the same time in few cases our effort becomes futile. That is the reason why the robotic arm has been introduced. There exist various kinds of robotic arms. It has been really successful. The Amazon Robotic Challenge (ARC) was introduced by
Amazon Robotics in 2015 to stimulate research into automation and importantly robotic manipulation for picking and stocking of products. There has been the use of sensors for the robotic arm but it was not accurate. The most safest and easiest is the use of camera which is so called as visually controlled robotic arm. But as we know that most of the visionrelated control strategy in robotics, there is no exact goal image or goal pose to achieve. Instead, we target to recover visibility of the target as soon as possible on any view or pose for any purpose. The major focus in vision planned robotics is on maintaining the aim within the camera field of view (FoV). In the use of bomb diffusion and for further purposes the use of this camera controlled robotic arm has been successful. The main reason behind its success is the safety of our health. The enhancement of an brightly viewed control system for a robotic user that it is guided by the user's mind. The goal objects are detected by a vision system and then viewed by the user in a video that shows them fused with flicking diamonds that are designed to excite electroencephalograph (EEG) signals at different frequency levels. Through the research of the invoked EEG signals, a brain computer interface (BCI) is developed to infer the exact object that is required by the users which are then transferred to the shared control system, where it is enabled by visual serving (VS) techniques to achieve accurate object manipulation.

\section{WHY DO WE GO FOR A ROBOTIC ARM}

A robotic arm is a type of machine like arm, which can be programmable, that has similar functions to that of a human arm. The arm may be the complete 
work of the mechanism or may be just a small part of a more composite robot. The links of such a controller are connected by joints like those of the humans joints allowing either rotational motion (such as in a jointed robot) or translational (linear) movement. The joints of the manipulator can be considered to form a activity chain. The end of the kinematic chain of the robotic arm is called the end effector and it is similar to the human hand. The end effectors, or robotic arm, can be designed to perform any desired task or we can say multitasking can be done such as welding, gripping, chemical related activities, spinning, picking up items etc., depending on the application. For example, robotic arms in various situations perform a variety of tasks such as welding and not only welding but also few rotations and displacement during assembly. In few situations, close production of the human hand (arm) is very much desired and needed, because the robots are designed to conduct and control bomb diffusion and disposal.

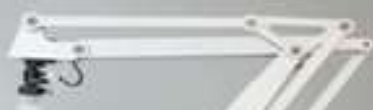

Figure 1: Robotic arm

A Robotic arm is a machine that copies or imitates the activity of a human hand. Machine arms are combined forms of multiple paths connected by joints powered by actuators. One end of the arm is attached to a steady base while the other end has a tool, which can be controlled by humans directly or from a distance. A machine- designed and controlled arm is called a robotic arm. However, a robotic arm is just one of different kinds of different machine arms. Mechanical arms can be as simple as pupeeters or as complex and difficult as artificial arms. We can also sum it up as, if a mechanism can take an object, hold the object, and then transfer it just like a human arm, then it can be categorized as a robotic arm. Recent evolvements have been brought that leads to future development in the medical field with important and with the robotic arm in general. The arm build by the mechanical engineers, their only goal is for the arm to perform a task that cannot be performed by any ordinary human arms

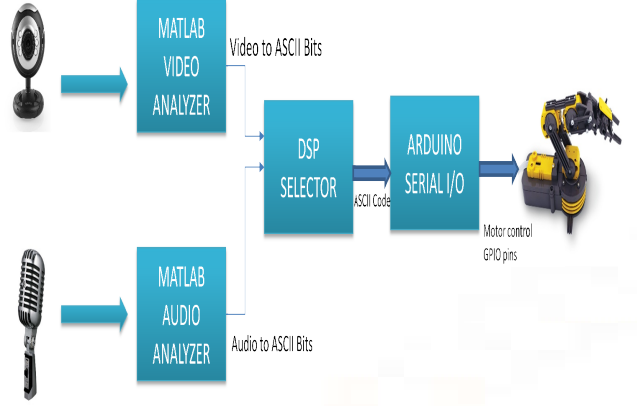

Figure 2: Block diagram

The development of cars would have been extremely difficult if the machine arms were not introduced. This problem was brought up with a solution in 1962 when the first machine arm was used in a "General Motors" factory. Use of this machine arm, also known as an machine robot, engineers achieved and accomplished difficult tasks. In addition to this, the removal of die-castings was one of the major steps in improving the abilities of a mechanical arm. With the involvement of such developed technology, mechanics were able to easily remove unnecessary metal while working the mold cavities. That is how, welding became increasingly popular for machine arms. ${ }^{[5]}$

Surgical arms were also developed. It was first used in 1985 when a neurosurgical biopsy was conducted. Although 1985 was the first time a surgery actually took place with a machine arm, scientists have been working on developing surgical arms for years. Complex robotic arms were developed and used for surgical purposed in 1495 which was originally developed by Da Vinci.

\section{SYSTEM OVERVIEW}

In the proposed model, the use of camera is introduced. In this model, the expenditure is less compared to the existing model also it makes our tasks easier. Instead of the IR sensors, the robotic Arm observes/captures the human mobility virtually through a webcam and works as per our instruction, because of which the accuracy is more.

There are various aspects that has been identified beneficial to characterize robotic systems modularity vs. integration, computation vs. embodiment, scheduling vs. review, or generality vs. assumptions. The dichotomy scheduling vs. review in robotics represents only two (important) classes in the spectrum of solutions. These range from open-loop solutions that exploit assumptions and knowledge 
about the task and the workspace at design time, to feedback strategies that use runtime information to drive robot's activities with uncertainties.

\section{A. CONTROL OF ROBOTIC ARM THROUGH RELAY}

A relay is typically an electrically operated switch. Electromagnets were used by many relays to operate a switch, but other working mechanisms are also applied, including the solid-state relays. Relays are used so that we can operate a circuit by a unique lowpower signal, or where many circuits were controlled by one signal. Long distance telegraph circuits as amplifiers used the first relays. The incoming signals from multiple circuits are re- transmitted to several circuitsWhen the current is switched off, the contacts gets opened, switching the circuit off. A beneficial characteristic of relays is that the circuit powering the coil is completely detached from the circuit switched on by the relay. This is the reason why relays were used where a high voltage circuit is controlled by a safe low-voltage circuit.

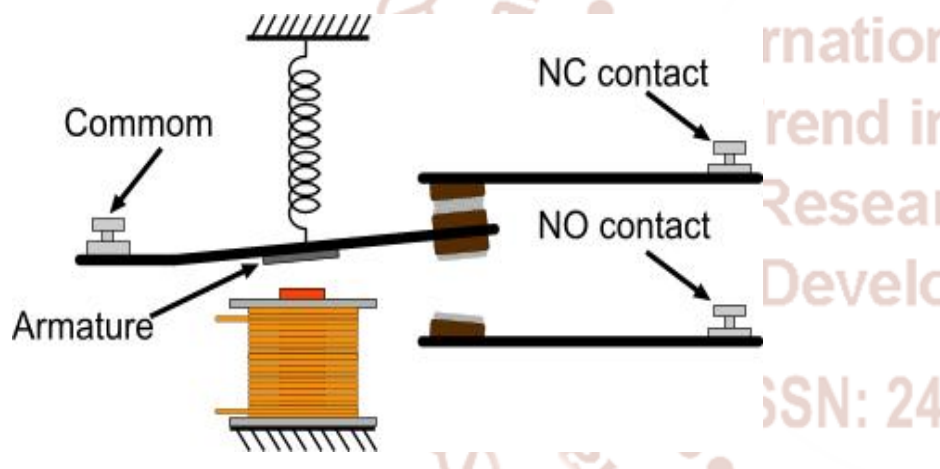

Figure 3: Working of Relay

\section{B. ESTIMATION OF MOTION:}

Motion detection is the method of detecting the movement vectors that explains the conversion from one 2D image to another which is usually from sequence frames in a video format. It is an ill-posed problem because the movement is in three dimensions but the images are a projection of the $3 \mathrm{D}$ plane onto a 2D plane. The motion vectors may relate to the entire image (global motion estimation) or particular parts, like rectangular blocks, arbitrary shaped patches or even per pixel. The motion vectors may be depicted by a translational model or many other models that can precise the movement of a real video camera, such as rotation and translation in all three dimensions and zoom.

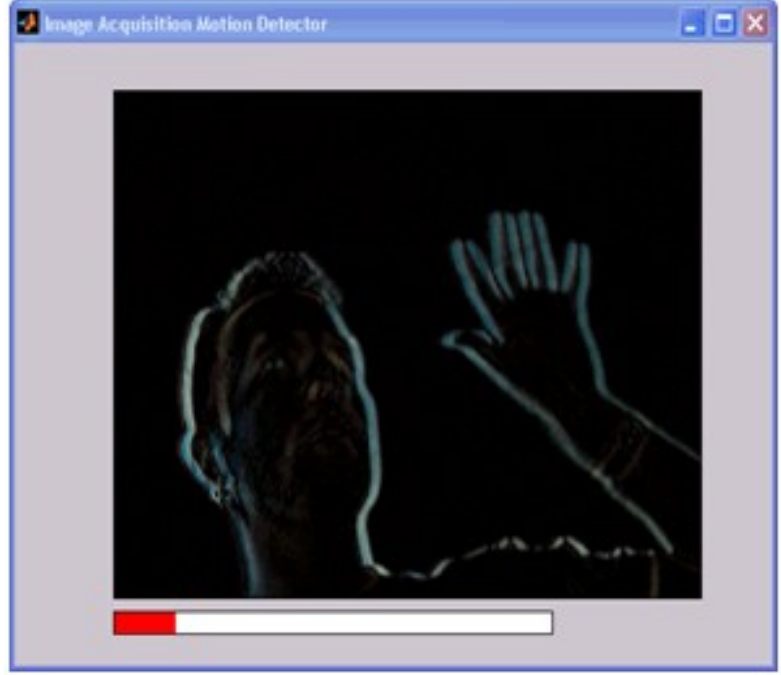

Figure 4: Motion Detection and Estimation

While the motion vectors are applied to an image to capture that transform to the next image is called motion compensation. As a method of capitalizing temporary redundancy, motion estimation and compensation are key sections of video minimization. Most of the video coding mechanisms use motion detection and substitution based on blocks that includes the MPEG series and the most lately HEVC.

\section{INTERFACE OF CAMERA ARDUINO:}

To verify the effectiveness of the adaptive algorithm, we designed the experiments. We designed three trajectories as the motions of a solid-colored object. In each trajectory, we captured 100 images to record the motion of the object. Then, we used the algorithm with and without the adaptive algorithm to track the object in all 100 images. We then calculated the number of the images that had been tracked successfully.

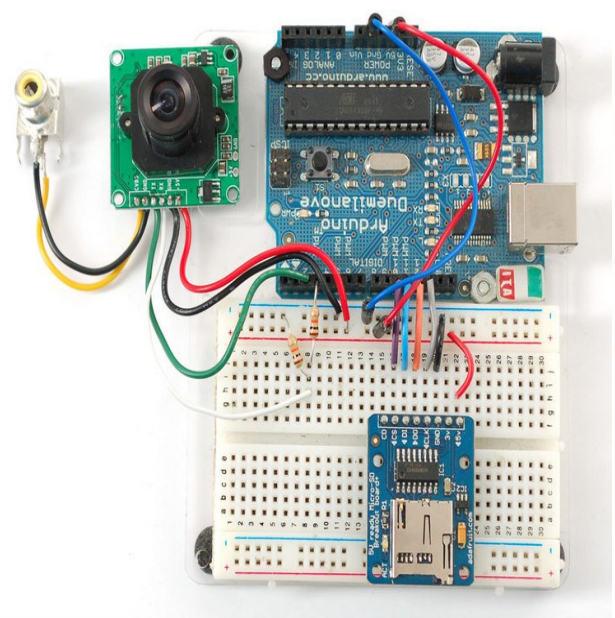

Figure 5: Camera Interface 
This camera module can perform image processing such as AWB (auto white balance), AE (automatic exposure) and AGC (automatic gain control), for the video signal coming from CMOS sensor. What's more, in fusion of other advanced technology such as image enhancement processing under low illumination, and image noise intelligent forecast and suppress, this module would output high quality digital video signals by standard interface. built-in JPEG decoder supported real time encoding for collected image, and external controller can easily read the $\mathrm{M}-\mathrm{JPEG}$ video streams, achieving the camera design of double stream. Supported motion detection and OSD display function of screen characters and pattern overlay, capable of selfdefining detection area and sensitivity.

\section{ROBOTIC MODULE:}

Most of the robots work in auto assembly lines, putting cars together. Robots can do a plenty of work in more efficient manner than human beings because of their accuracy. They always work in the exact manner at the same place, and also they have always tightened bolts with an equal amount of force, no matter how long they work. Designing such robots is also very necessary in the computer industry. It takes an incredibly precise hand to put together a tiny microchip.

The most commonly developed robot is the robotic arm. Generally the robotic arms are made up of seven metal parts which is joined by six joints. The computer monitors robot by rotating each and every step motors connected to each joint. Step motors rotate and move in exact increments unlike any other ordinary motors which allow the machine to move the arm very accurately, repeating the exact movement again and again. Motion sensors are used by the robots so that they move just the right amount.

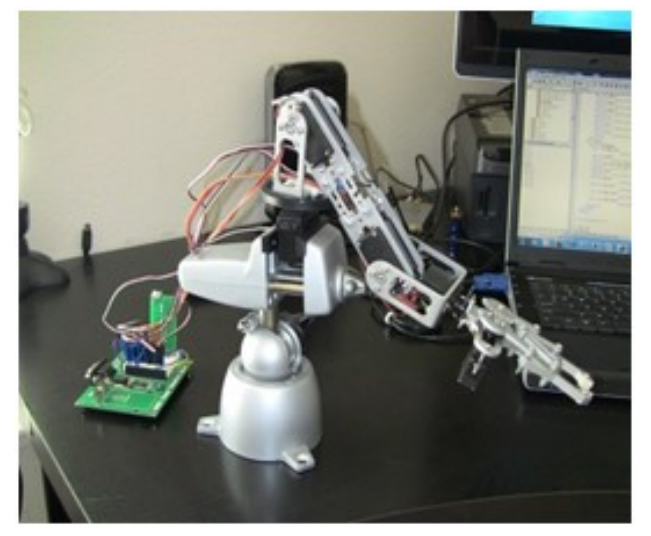

Figure 6: Working of Robotic Module

\section{CONCLUSION AND FUTURE WORK}

The experimental results indicated that the developed system could achieve high accuracy with various subjects. In future work, we will endeavor to improve the algorithms in the robotic system to improve its performance and then use them in real applications.

This efficient mapping would allow for an online multihypothesis tracking to track a potential occlude in advance and foresee an occlusion scenario.

\section{REFERENCES}

1. L. Torabi and K. Gupta, "An autonomous sixDOF eye-in-hand system for in situ 3D object modeling," Int. J. Robot. Res., vol. 31, no. 1, pp. 82-100,2012.

2. S. Radmard, D. Meger, E. A. Croft, and J. J. Little, "Overcoming unknown occlusions in eyein-hand visual search," in Proc. IEEE Int. Conf. Robot. Autom., 2013, pp. 3075-3082.

3. M. Rickert, A. Sieverling, and O. Brock, "Balancing exploration and exploitation in sampling-based motion planning," IEEE Trans. Robot., vol. 30, no. 6, pp. 1305-1317, Dec. 2014.

4. C. Yang, M. Fu, and H. Ma, Advanced Technologies in Modern Robotic Applications. Springer, Singapore, 2016.

5. T. Carlson and J. D. R. Millan, "Brain-controlled wheelchairs: A robotic architecture," IEEE Robotics \& Automation Magazine, vol. 20, no. 1, pp.65-73, 2013.

6. J. Zhao, W. Li, X. Mao, and M. Li, "SSVEPbased experimental procedure for brain-robot interaction with humanoid robots." Journal of Visualized Experiments, vol. 2015, no. 105, 2015.

7. Hajiloo, M. Keshmiri, W. F. Xie, and T. T.model predictive control for a constrained image-based visual servoing,'IEEE Transactions on Industrial Electronics, vol. 63, no. 4, pp. 2242-2250, 2016.

8. S. Ren, K. He, R. Girshick, and J. Sun, "Faster RCNN: Towards Real-Time Object Detection with Region Proposal Networks," IEEE Transactions on Pattern Analysis and Machine Intelligence, vol. 39, no. 6, pp. 1137-1149, June 2017.

9. N. Correll, K. E. Bekris, D. Berenson, O. Brock, A. Causo, K. Hauser,K. Okada, A. Rodriguez, J. M. Romano, and P. R. Wurman, "Analysis and Observations From the First Amazon Picking 
Challenge," IEEE Transactions on Automation

Science and Engineering, vol. PP, no. 99, pp. 117, 2017.

10. C. Hern'andez, M. Bharatheesha, W. Ko, H. Gaiser, J. Tan, K. van Deurzen, M. de Vries, B. V. Mil, J. van Egmond, R. Burger, M. Morariu, J. Ju, X. Gerrmann, R. Ensing, J. van Frankenhuyzen, and M. Wisse,

11. Team Delft's Robot Winner of the Amazon Picking Challenge 2016, In S. Behnke, R. Sheh, S. Sariel, and D. D. Lee, eds., RoboCup 2016 Proceedings (to appear), volume 9776 of Lecture Notes in Computer Science, pp. 613-624, Springer, 2017.

12. J. Mahler and K. Goldberg, "Learning DeepPolicies for Robot Picking by Simulating Robust Grasping Sequences," in Proceedingsof the 1st Annual Conference on Robot Learning, ser. Proceedings ofMachine Learning Research, S. Levine, V. Vanhoucke, and K. Goldberg,Eds., vol. 78. PMLR, 13-15 Nov 2017, pp. 515-524.

13. S. Karaman and E. Frazzoli," "Sampling-based algorithms for optimalmotion planning," Int. J. Robot. Res., vol. 30, no. 7, pp. 846-894, 2011.

14. Y. Yu and K. Gupta, "C-space Entropy: A measure for view planning andexploration for general robot-sensor systems in unknown environments,'Int. J. Robot. Res., vol. 23, no. 12, pp. 1197-1223, 2004.

15. N. R. Gans, G. Hu, K. Nagarajan, and W. E. Dixon, "Keeping multiplemoving targets in the field of view of a mobile camera," IEEE Trans.Robot., vol. 27, no. 4, pp. 822-828, Aug. 2011.

16. H. Liu, "A fuzzy qualitative framework for connecting robot qualitative and quantitative representations," IEEE Transactions on Fuzzy Systems, vol. 16, no. 6, pp. 1522-1530, 2009.

17. K. Tarabanis, R. Y. Tsai, and A. Kaul, "Computing occlusion-free viewpoints," IEEE Trans. Pattern Anal. Mach. Intell., vol. 18, no. 3, pp. 279-292, Mar. 1996. 